\title{
Blood Group Antigens and Integrins as Biomarkers in Head and Neck Cancer: Is Aberrant Tyrosine Phosphorylation the Cause of Altered $\alpha 6 \beta 4$ Integrin Expression?
}

\author{
Thomas E. Carey, PhD $^{1}$, Thankam S. Nair, MS ${ }^{1}$, Catherine Chern, $\mathbf{B S}^{1}$, \\ Monica Liebert, $\mathbf{P h D}^{2}$, H. Barton Grossman, $\mathbf{M D}^{2}$, Gregory T. Wolf, $\mathbf{M D}^{1}$, and \\ Carter Van Waes, MD, $\mathbf{P h D}^{1}$ \\ 1 Laboratory of Cancer Biology, Department of Otolaryngology/Head and Neck Surgery, \\ University of Michigan Cancer Center, Ann Arbor MI 48109-0506 \\ 2 Urologic Oncology Laboratory (HBG, ML), Department of Surgery, University of \\ Michigan Cancer Center, Ann Arbor MI 48109-0506
}

\begin{abstract}
Head and neck cancer is a capricious disease that varies greatly in its clinical behavior. The development of biomarkers that can distinguish between biologically aggressive and indolent tumors has been a long term goal of our laboratories. Predictive markers applicable to biopsy specimens should facilitate clinical management through early identification of patients at greatest risk for early relapse or metastatic spread. Two prominent cell surface markers that we identified by raising monoclonal antibodies to squamous cell carcinomas are blood group antigens and the $\mathrm{A} 9$ antigen/ $\alpha 6 \beta 4$ integrin. Both of these markers are abnormally displayed in squamous cancers of the head and neck and serve as indicators of early relapse. Loss of blood group antigen expression is a stronger single indicator than is overexpression of the $\alpha 6 \beta 4$ integrin. However, use of both markers together is a stronger predictive indicator than is either alone. We know little about the function of the blood group antigens in squamous cells except that the mature antigens are associated with differentiation. Similarly, the function of the $\alpha 6 \beta 4$ integrin is also not fully understood. Integrin $\alpha 6 \beta 4$ is thought to serve as an extracellular matrix receptor, but its ligand has not been confirmed. In resting epithelium, the $\alpha 6 \beta 4$ integrin is polarized to the basal aspect of the basal cell as a component of the hemidesmosome, the anchoring structures of the epithelia. This basal polarization is lost in migrating normal squamous cells and squamous carcinomas. Tyrosine phosphorylation of the $\beta 4$ subunit is absent or greatly reduced in malignant cells and this may be a critical signal for subcellular localization of $\alpha 6 \beta 4$ and cell anchoring. On the basis of our current experimental results, we postulate that tyrosine phosphorylation of the $\beta 4$ subunit is a reversible signal that regulates cell migration in normal and malignant cells, and may therefore be an important initial event in the metastatic cascade.

(C) 1993 Wiley-Liss, Inc.
\end{abstract}

Key words: squamous cell carcinoma, $\mathrm{ABH}$ antigens, H-type- 2 antigen, $\alpha 6 \beta 4$ integrin, prognosis, protein tyrosine phosphatase, hemidesmosomes

Address correspondence to Dr. Thomas E. Carey, Laboratory of Head and Neck Cancer Biology, $6020 \mathrm{KHRI}$, The University of Michigan, 1301 East Ann Street, Ann Arbor, MI 48109-0506.
Abnormal expression of normal antigens is a characteristic of squamous carcinomas. We raised monoclonal antibodies against cell lines cultured from head and neck squamous carcinomas which identify two epithelial antigenic markers that are prominently and abnormally expressed. These antibodies, UM-G10 and 
UM-A9, define the H-type-2 blood group antigen [1] and the $\alpha 6 \beta 4$ integrin, respectively [2,3]. The H-type- 2 antigen is a carbohydrate structure that serves as the acceptor site for the $\mathrm{A}$ and B blood group antigens. In the epithelium, the H-type-2 antigen is normally expressed only on the basal epithelial cells of persons who are either blood group A or B, but it is expressed throughout the epithelium of blood group $\mathrm{O}$ individuals. In many cases, squamous carcinomas express this antigen strongly even if the donor is blood type A or B $[1,4,5]$. In fact, overexpression of the $\mathrm{H}$-type-2 precursor in tumors often goes hand-in-hand with absence of the mature form. The antigen defined by the UM-A9 antibody is also strongly expressed by most squamous carcinomas even though this marker is more restricted in its expression in normal epithelium, where it is present only on the basal surface of the basal cells. Examples of the A9 antigen and blood group A antigen distribution in normal tongue and in an early invasive squamous cancer in a blood type $\mathrm{A}$ patient are shown in Figure 1.

Clues that these structures might serve as biomarkers of aggressive malignant behavior came initially from studies of cell lines cultured from primary and metastatic tumors in the same individuals, where the expression of the H-type-2 antigen $[1,6]$ and the A9 antigen $[2,6]$ were both more prominent on the metastatic or recurrent tumor cell lines than on the primary tumor cell lines. Furthermore, loss of blood group antigen expression had been shown to be
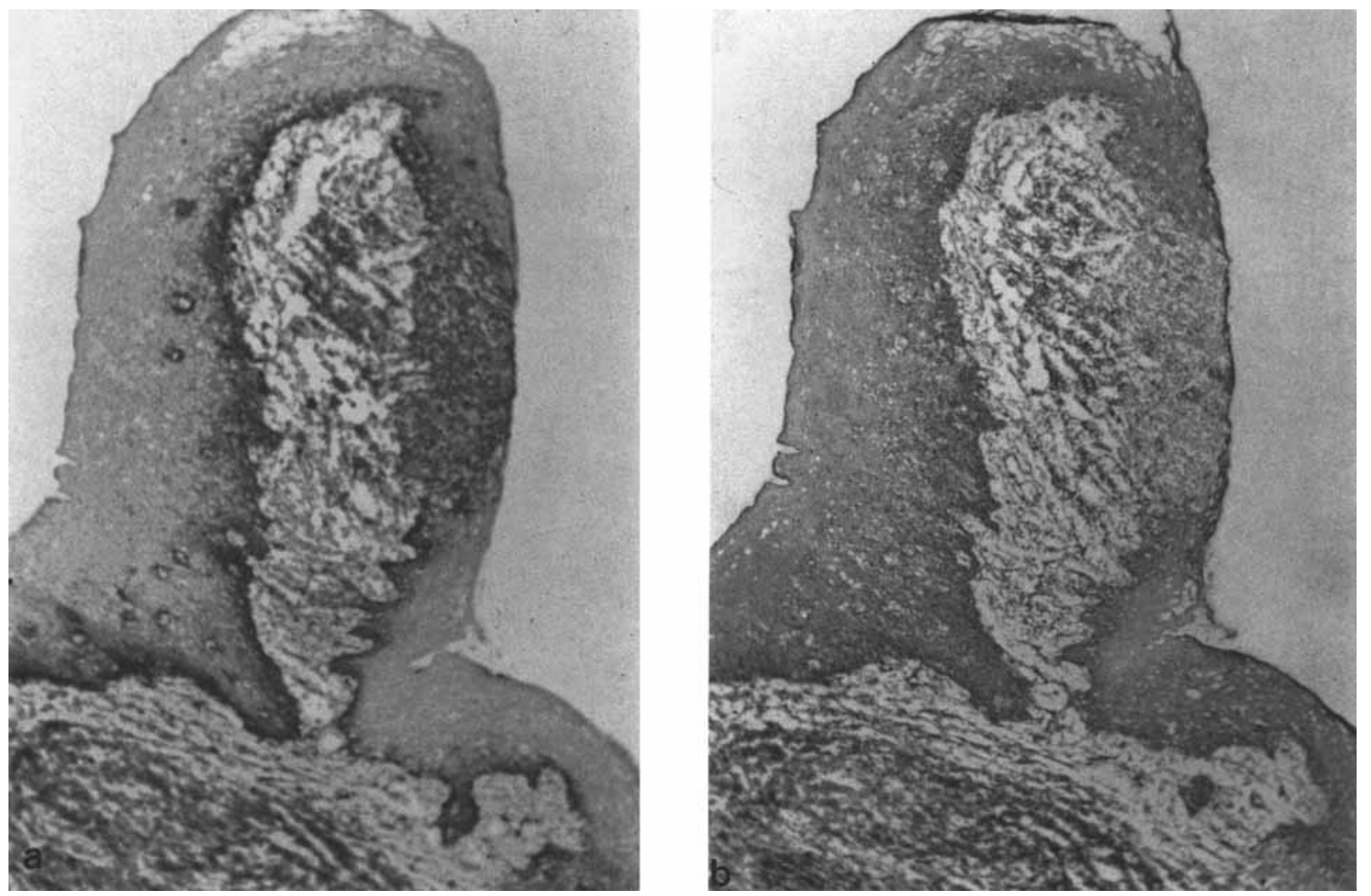

Fig. 1. Photomicrographs of immunoperoxidase stained frozen sections of tongue tissue from a patient with early invasive squamous cell cancer. (a) Section stained with UM-A9 antibody to the $\alpha 6 \beta 4$ integrin. Note that $\alpha 6 \beta 4$ integrin expression is limited to basal epithelial cells in the normal tissue but that the tumor is stained from its epithelial surface to its invasive edge. (b) Adjacent section stained with a monoclonal antibody specific for blood group $A$. Note that in this section blood group $A$ is expressed throughout the normal epithelium but is absent in the region of the tumor. (Original magnification $40 \times$ ) Reprinted from Carey et al. [4] with permission of Mosby Year Book, Inc. 
associated with metastasis in cervical carcinoma [7] and invasive behavior in bladder cancer [8]. We analyzed blood group and A9 antigen expression in a series of tumors from patients with previously untreated head and neck cancer and reported that overexpression of A9 antigen and loss of mature blood group antigens were each associated with early relapse $[4,5]$. When we performed a subsequent analysis with longer follow-up in the same group of head and neck cancer patients [9], we found that as a single variable, loss of blood group antigen is a stronger predictive marker for shortened, disease-free survival (Fig. 2) than is overexpression of A9 antigen/ $\alpha 6 \beta 4$ integrin. However, when both variables are combined it is possible to separate patients into three prognostic groups (Fig. 3). These observations have led to several important research questions: Why are these markers associated with biological behavior in vivo? How can we best use these markers clinically? Can they be used for selecting patients for therapy? Can they be used to monitor response to chemopreventive therapy? The answers to these ques- tions may become clear once the functional significance of the altered antigen expression is known.

Very little is known about the function of the blood group antigens in epithelia, making it difficult to determine why these are strong prognostic markers. One possibility is that as squamous epithelial cells mature and differentiate, they display the mature blood group antigen on the cell surface. Thus, failure to express mature blood group antigens may indicate failed maturation and a relatively undifferentiated phenotype. An alternative hypothesis is that blood group antigens are part of cell-to-cell adhesion structures and loss of these structures facilitates metastatic behavior. Since altered expression of blood group antigens and association of aberrant expression with prognosis has been noted by several investigators, including the recent report by Lee et al. [10] that expression of blood group A antigen in lung tumors is a favorable prognostic indicator in non-small cell lung cancer, it is clear that much more work on this subject is indicated.

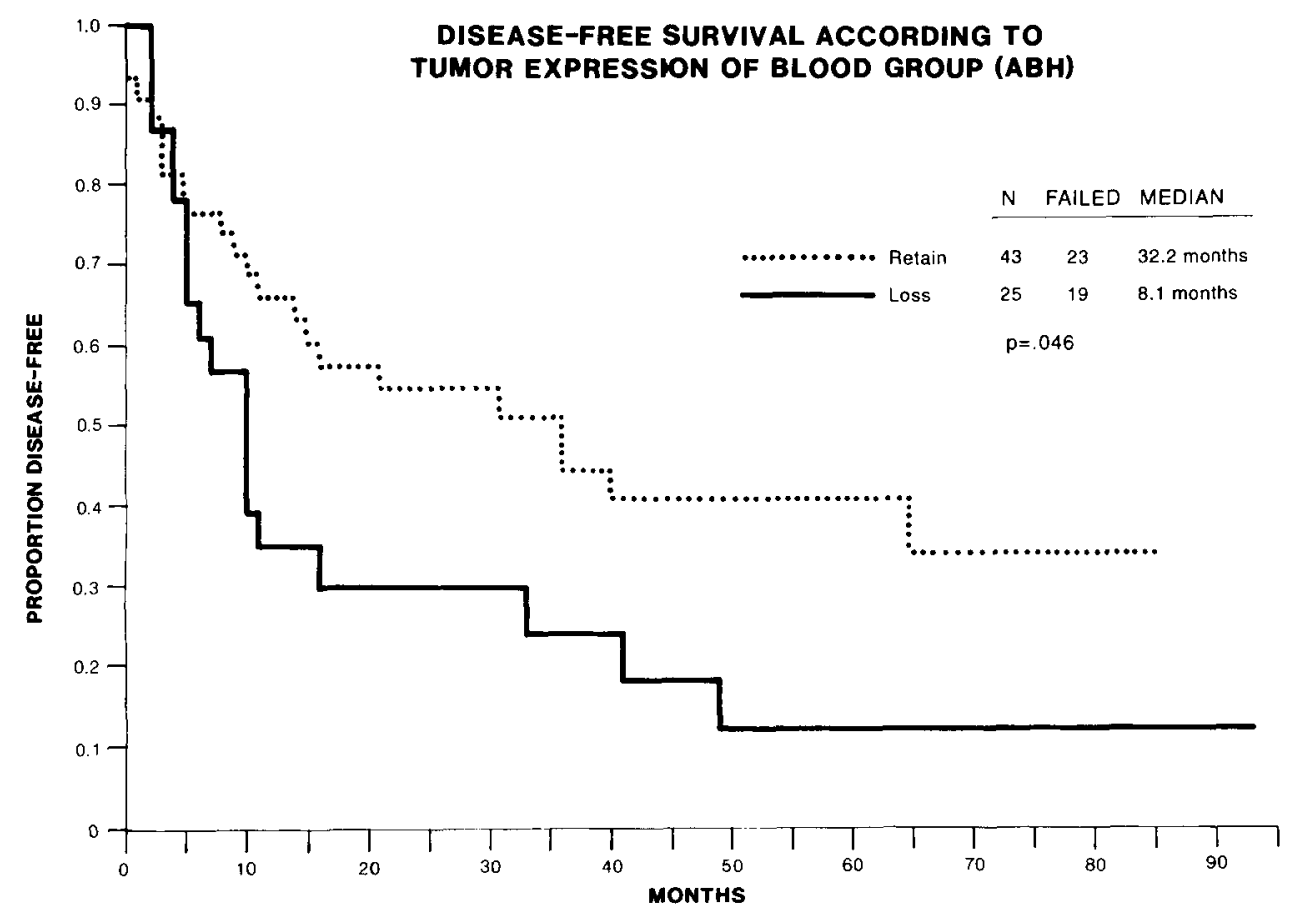

Fig. 2. Disease-free survival according to tumor expression of $A B H$ blood group. Disease-free interval was significantly longer in patients whose tumor retained blood group expression compared to those who had lost expression. Reprinted from Wolf et al. [9]. 


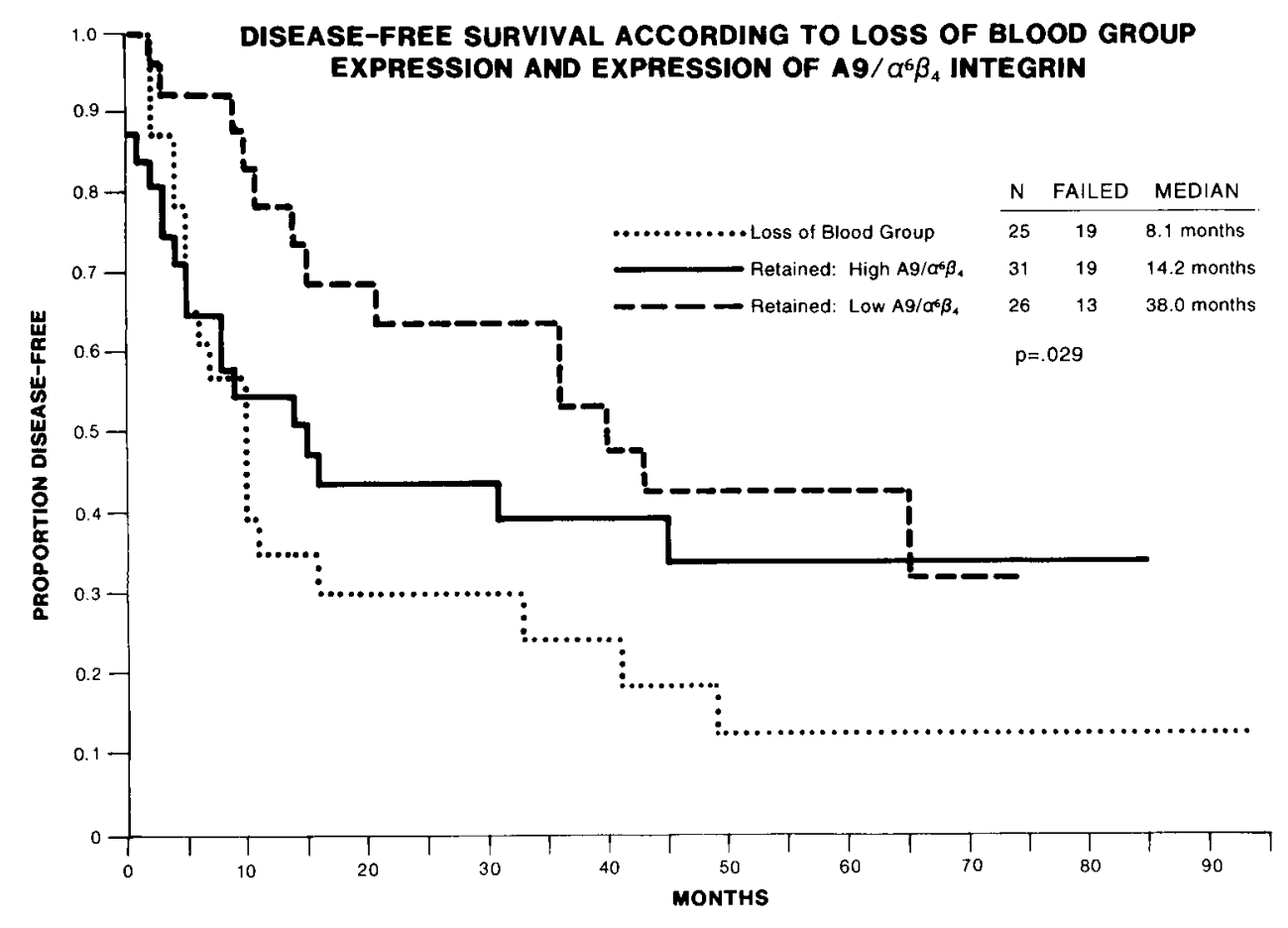

Fig. 3. Disease-free survival according to intensity of $A 9 / \alpha 6 \beta 4$ integrin expression and loss of blood group $(\mathrm{ABH})$ expression in the patients' tumors. The longest median survival was associated with tumors exhibiting low A9/ $\alpha 6 \beta 4$ expression and retained blood group expression. Reprinted from Wolf et al. [9].

\section{$\alpha 6$ AND $\beta 4$ INTEGRIN SUBUNIT EXPRESSION IN MALIGNANT CELLS}

Van Waes et al. [3] showed by immunoprecipitation and preclearing experiments that the A9 antigen was a heterodimeric complex of two protein chains, an alpha subunit $(\alpha 6)$ and a beta subunit ( $\beta 4)$, typical of the integrin family of cell surface receptors. Overexpression of this integrin appears to be a common factor in several epithelial cancers since it was discovered independently as a tumor antigen by several groups who raised antibodies against other tumors. For example, Kennel and co-workers [11] described the TSP-180 antigen in murine lung carcinoma, a marker that is overexpressed on metastatic but not non-metastatic clones of the Lewis lung carcinoma cell line. TSP-180 was later shown to be the $\alpha 6 \beta 4$ integrin [12]. These authors also demonstrated that human lung carcinomas exhibit aberrant expression of the $\alpha 6 \beta 4$ integrin [13]. Similarly, Kajiji et al. [14] raised antibodies against pancreatic carcinomas that identify the $\alpha 6 \beta 4$ integrin. Thus, the $\alpha 6 \beta 4$ integrin is a prominent immunogenic determinant on many epithelial cancers.

In our own group, the BQ16 monoclonal antibody was raised against human bladder carcinoma cells [15]. UM-A9 and BQ16 showed strong similarities in the staining of normal epithelia, but differed in their reactivity with certain bladder cancer cell lines. At first we felt that these antibodies identified different structures, until one of us (ML) suggested that the antibodies might identify different subunits of the $\alpha 6 \beta 4$ integrin. If so, tumors that were positive for BQ16 but negative for UM-A9 reactivity might express only one of the subunits. In fact, this hypothesis was correct. BQ16 identifies the $\alpha 6$ subunit and UM-A9 identifies either the $\beta 4$ subunit alone or the intact $\alpha 6 \beta 4$ complex [15]. Since it appears that $\beta 4$ is expressed only in a complex with $\alpha 6$, it is difficult to rule out the possibility that the UM-A9 epitope is determined by both chains. An experiment that compares the two antibodies for their ability to 


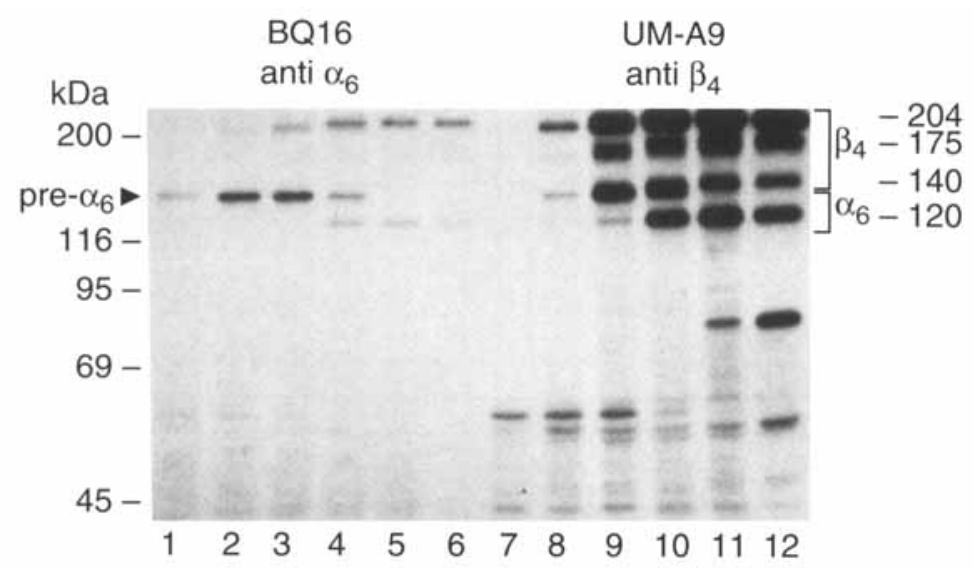

Fig. 4. Pulse-chase immunoprecipitation experiment comparing proteins precipitated from the same cell extracts by monoclonal antibodies UM-A9 and BQ16. UM-SCC-38 cells were labeled with ${ }^{35} \mathrm{~S}$-methionine for 15 or $30 \mathrm{~min}$ and then either lysed or refed with medium containing cold methionine and incubated for additional periods of time ranging from $30 \mathrm{~min}$ to $24 \mathrm{hr}$. The labeled cells were lysed and immunoprecipitated with either UM-A9 or BQ16 and protein-A sepharose beads. The immunoprecipitates were washed several times and then boiled in SDS buffer under reducing conditions, separated by polyacrylamide gel electrophoresis, and analyzed by autoradiography. Labeling conditions: 15 min pulse only-lanes 1,$7 ; 30$ min pulse only-lanes 2,8 ; $30 \mathrm{~min}$ pulse plus $30 \mathrm{~min}$ cold chase-lanes 3,$9 ; 1 \mathrm{hr}$ cold chase-lanes 4,$10 ; 4 \mathrm{hr}$ cold chase-lanes 5,$11 ; 24 \mathrm{hr}$ cold chase-lanes 6,12 . (Reproduced from [15], with permission of the publisher.)

21]. This experiment supports the hypothesis that BQ16 binds to the $\alpha 6$ subunit and that UM-A9 binds either to the $\beta 4$ subunit or the intact $\alpha 6 \beta 4$ heterodimer. Since the $\alpha 6$ subunit can also combine with the $\beta 1$ subunit, those bladder carcinoma cell lines that are positive for BQ16 staining and negative for UM-A9 staining most likely express the $\alpha 6 \beta 1$ integrin but not the $\alpha 6 \beta 4$ integrin. The functional significance of this difference in integrin subunit expression is not yet known. Thus far we have not observed any examples of squamous cancers that are $\alpha 6$ positive and $\beta 4$ negative.

\section{$\alpha 6$ AND $\beta 4$ INTEGRIN EXPRESSION IN NORMAL EPITHELIUM}

On normal epithelia, BQ16 and UM-A9 have identical staining patterns along the basal surface of skin, mucosa, and transitional epithelia (Fig. 5). In Figure 5, the same section of normal skin is co-stained with UM-A9 and BQ16. Since UM-A9 is an IgG2a antibody and BQ16 is an IgG1 antibody, the binding could be detected with one secondary antibody specific for IgG2a, labeled with fluorescein, and another antibody specific for IgG1 labeled with rhodamine. 

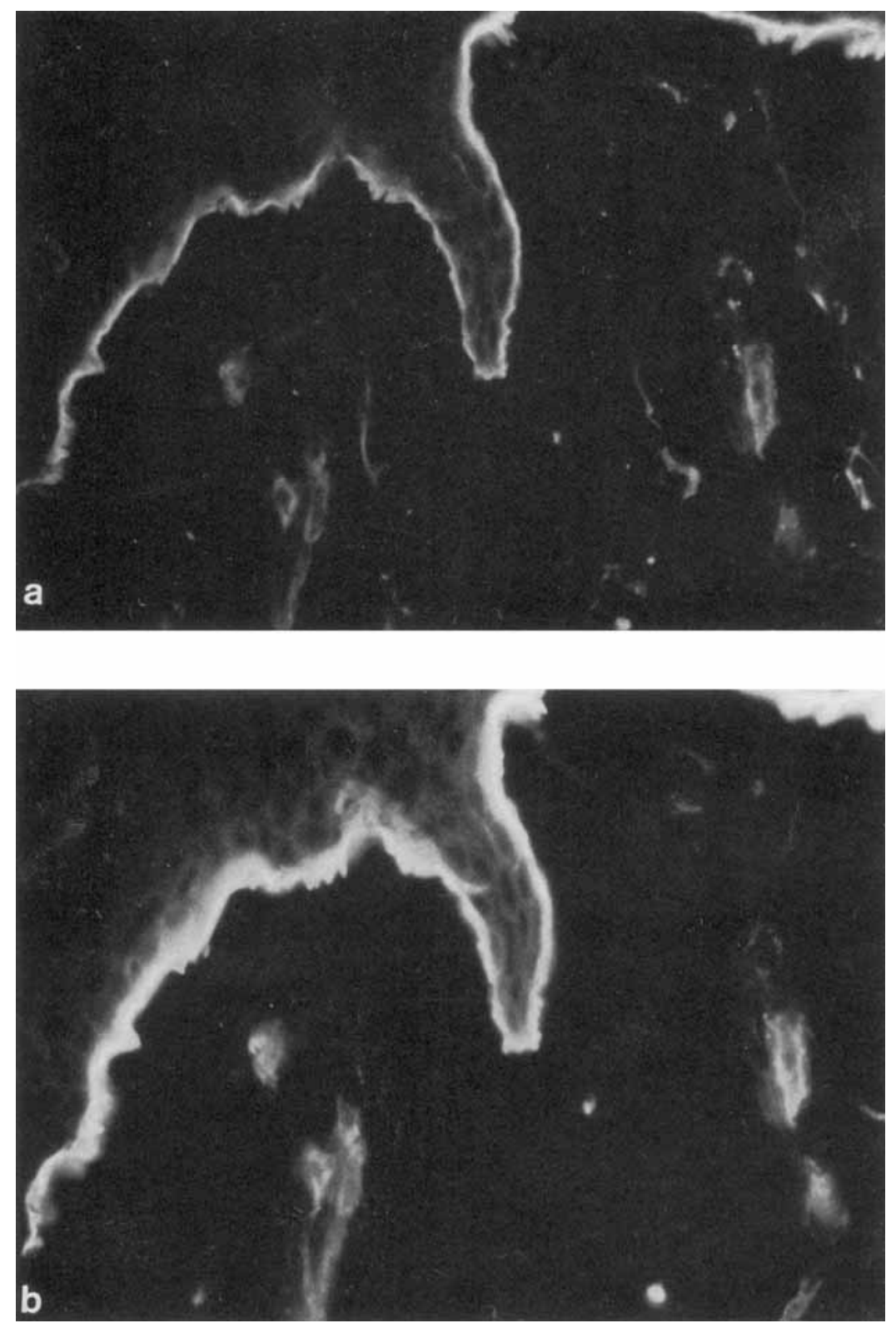

Fig. 5. Immunofluorescence staining of frozen sections of normal skin with UM-A9 and BQ16 antibodies. The section was incubated with a mixture of UM-A9 and BQ16, washed and incubated with a mixture of secondary antibodies to IgG2a (labeled with fluorescein) and IgG1 (labeled with rhodamine). The sections were evaluated for fluorescence under a $50 \times$ objective. The same field was photographed twice, once with a filter for green fluorescence and once with a filter for red fluorescence. (a) Linear staining of the basal surface of the epithelium by UM-A9. Note also staining of some small vessels and nerve fibers in the dermis. (b) Linear staining of the basal surface of the epithelium by BQ16. Note that this antibody also stains small vessels in the dermis. 


\section{ROLE OF $\alpha 6 \beta 4$ INTEGRIN IN THE ANCHORING PROCESS}

The location of $\alpha 6 \beta 4$ along the basement membrane suggests that this integrin is involved in the anchoring process in normal epithelia. Ultrastructural electron microscopic studies by several groups [16-18] have shown that the $\alpha 6 \beta 4$ integrin is clustered in the region of the hemidesmosome in normal epithelia, offering further support for the concept that $\alpha 6 \beta 4$ is involved in the anchoring process. However, expression of the $\alpha 6 \beta 4$ integrin is depolarized $[19,20]$ in tumors and in normal keratinocytes growing and migrating under wound healing conditions in which hemidesmosomes do not form until migration is completed [19]. An example of the peripheral non-basal distribution of the $\alpha 6 \beta 4$ integrin in normal keratinocytes grown in basal keratinocyte medium, a condition that fosters cell migration, and to some extent, mimics the wound healing response, is shown in Figure 6a. Basal polarization of the integrin occurs in normal keratinocytes grown in the same basal keratinocyte medium to which $1.4 \mathrm{mM}$ calcium and $10^{-8} \mathrm{M}$ retinoic acid were added, as shown in Figure 6b. The Swisscheese pattern of $\alpha 6 \beta 4$ integrin expression noted under these conditions is visually similar to the staining pattern observed with antibodies to

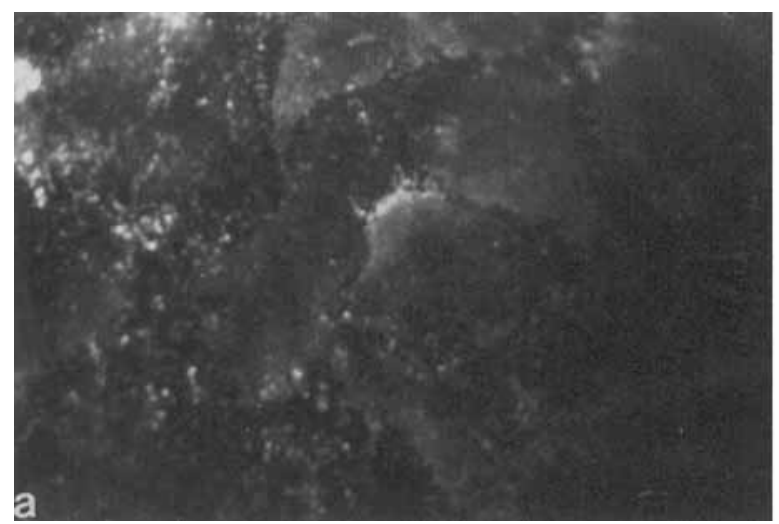

other hemidesmosomal components in the hemidesmosome-forming $804 \mathrm{G}$ cell line [21]. Based on these and other observations, we postulate that the $\alpha 6 \beta 4$ integrin serves two roles, one in migration where high expression is accompanied by peripheral distribution, and a second in anchoring where the integrin becomes localized to the basal surface as it is in normal epithelia. The mechanisms that control the distribution of the $\alpha 6 \beta 4$ integrin on the cell surface are not known; one possibility is that changes in the phosphorylation state of the $\beta$ subunit may be important [20].

\section{TYROSINE PHOSPHORYLATION OF THE B4 SUBUNIT}

Falcioni et al. [22] reported that the $\beta$ subunit of $\alpha 6 \beta 4$ integrin is phosphorylated on serine, threonine and tyrosine residues in murine Lewis lung carcinoma. The amino acid sequence of the cytoplasmic domain of human $\beta 4$ protein contains numerous consensus phosphoserine and phosphothreonine acceptor sites, as well as a single phosphotyrosine site. We previously reported that the degree of phosphorylation of the $\beta 4$ subunit varied with culture conditions [20], and that the incorporation of inorganic phosphate into $\beta 4$ protein increased under culture conditions associated with induction of

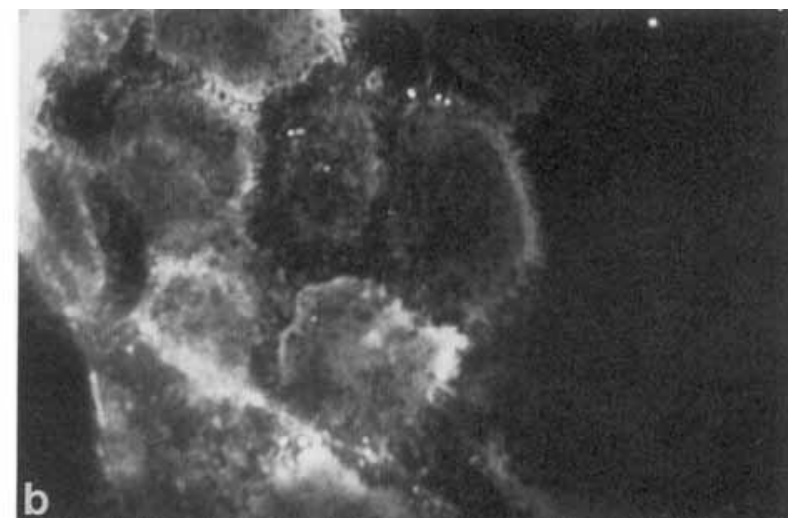

Fig. 6. Immunofluorescence photomicrographs of normal keratinocytes growing in vitro and stained with the UM-A9 antibody. (a) Keratinocytes grown in basal keratinocyte medium without epidermal growth factor. Note that the $\alpha 6 \beta 4$ integrin is distributed on the peripheral margins of the cells and in bright punctate spots on microfilopodia in these migrating cells. (b) Keratinocytes grown in basal keratinocyte medium to which $1 \mu \mathrm{M}$ retinoic acid and $1.4 \mathrm{mM}$ calcium chloride had been added $24 \mathrm{hr}$ previously. In this culture the cells have migrated into colonies and the $\alpha 6 \beta 4$ integrin expression is now mostly polarized to the basal surface in a "Swiss cheese" pattern. 
keratinocyte differentiation, and decreased in the presence of retinoic acid, which stimulates cell migration and DNA synthesis in the absence of calcium. Our initial phosphoamino acid analysis experiments revealed only phosphoserine and phosphothreonine modifications on $\beta 4$. As there is only one consensus phosphotyrosine site, it is likely that this would be more difficult to detect, especially since it might be labile to protein tyrosine phosphatase cleavage. To examine tyrosine phosphorylation of the $\beta 4$ integrin, we used the protein tyrosine phosphatase inhibitor sodium orthovanadate and western blotting with antiphosphotyrosine antibodies. Normal cells and tumor cells were treated with $100 \mu \mathrm{M}$ vanadate for periods ranging from 0 to $24 \mathrm{hr}$. The cells were lysed, immunoprecipitated with UM-A9 antibody, subjected to electrophoresis, transferred to nitrocellulose, and probed with an antiphosphotyrosine antibody (Upstate Biotechnology Inc., Lake Placid, NY). No tyrosine phosphorylation was detected at $0 \mathrm{hr}$, but a doublet of phosphotyrosine-positive bands corresponding to the $205 \mathrm{kD} \beta 4$ protein and a higher molecular weight band of $\sim 230 \mathrm{kD}$ was detected in the $\alpha 6 \beta 4$ immunoprecipitates from normal keratinocytes treated with vanadate for $24 \mathrm{hr}$. This signal was not present in lysates from identically treated UM-SCC-38 tumor cells (Fig. 7). Similarly, keratinocytes treated with vanadate undergo a spreading response (Fig. 8) that was not observed in the UM-SCC-38 cells under the same conditions. It also appears that vanadate-induced spreading may block the migration of keratinocytes into colonies typically induced in keratinocyte cultures by the addition of $1.4 \mathrm{mM}$ calcium as shown in Figure 8 .

The full significance of these observations is not known. Our current interpretation is that phosphorylation and dephosphorylation of tyrosine in the $\beta 4$ integrin subunit is a dynamic process in cultured keratinocytes. To observe a strong $\beta 4$ phosphorylation signal, it is necessary to block dephosphorylation with vanadate for $24 \mathrm{hr}$. This suggests that most of the $\beta 4$ protein is not phosphorylated on tyrosine in actively dividing and migrating cells. Treatment of normal cells with vanadate also results in inhibition of migration and induction of spreading, suggesting that tyrosine phosphorylation on $\beta 4$ and cessation of migration are related. In the UM-SCC-38 tumor cell cultures, $24 \mathrm{hr}$ of treat-

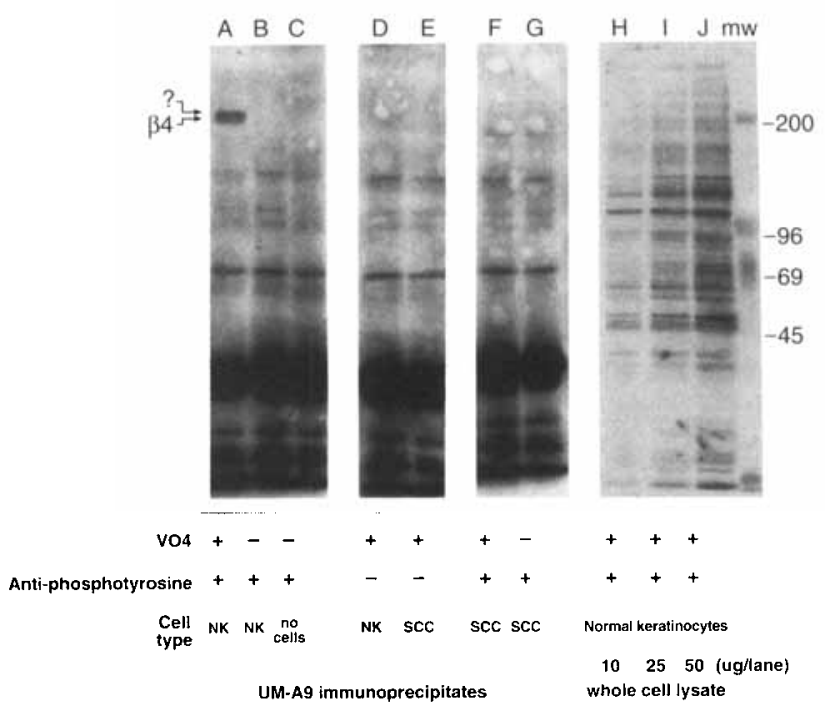

Fig. 7. Chemiluminescence staining with anti-phosphotyrosine antibody on a western blot of proteins immunoprecipitated from normal and malignant keratinocytes by UM-A9 antibody. Normal adult human keratinocytes and UM-SCC-38 cells were grown in keratinocyte growth medium until $24 \mathrm{hr}$ before harvest, when $100 \mu \mathrm{M}$ sodium orthovanadate was added to some cultures as indicated on the figure. The cells were lysed, and the $\alpha 6 \beta 4$ integrin was precipitated with UM-A9 antibody and protein-A sepharose beads after which the beads were washed, boiled in SDS reducing buffer, and the immunoprecipitates separated by polyacrylamide gel electrophoresis and transferred to nitrocellulose. The blots were incubated with antiphosphotyrosine antibody as indicated on the figure and developed using the enhanced chemiluminescence technique. Note that the only phosphotyrosine signal detected was a doublet at 205-230 kD corresponding to the $\beta 4$ protein and a slightly higher molecular weight band shown in lane $A$. The other bands are from secondary antibody binding to the reduced UM-A9 immunoglobulins as shown in lane $C$ where only the UM-A9 antibody and the protein A beads but no cell lysate were loaded. The same pattern was obtained in lanes $D$ and $E$ where no antiphosphotyrosine antibody was added to the blot. Lane $F$ shows that the tumor cells fail to phosphorylate $\beta 4$ on tyrosine. Lanes $\mathrm{H}$ $\mathrm{I}, \mathrm{J}$ are whole cell lysates showing the range of phosphotyrosine-modified proteins in normal keratinocytes.

ment with $100 \mu \mathrm{M}$ vanadate had no effect on either the morphology of the cells (not shown) or on the $\beta 4$ phosphotyrosine modification (Fig. 8). This suggests that failure to phosphorylate the $\beta 4$ tyrosine or rapid dephosphorylation of $\beta 4$, may correspond to the failure of tumor cells to stop migrating. The basis for this difference between normal and malignant cells is not known. Tumor cells may have an abnormality 


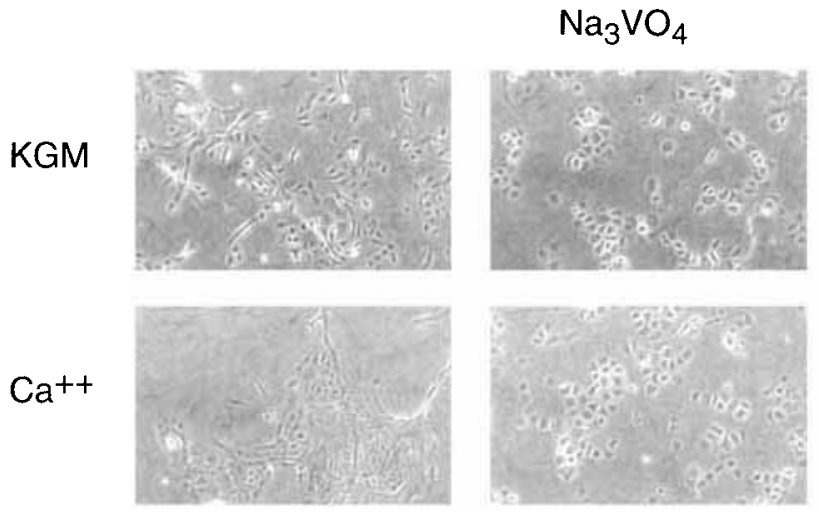

Fig. 8. Photomicrograph showing the effects of calcium and vanadate on normal keratinocyte cultures. Keratinocytes growing in complete keratinocyte growth medium were fed as indicated on the figure with either fresh medium, medium containing $100 \mu \mathrm{M} \mathrm{Na}_{3} \mathrm{VO}_{4}$, medium containing $1.4 \mathrm{mM} \mathrm{CaCl}{ }_{2}$, or both, $24 \mathrm{hr}$ before this photograph was taken. Note that cells fed with calcium migrate into colonies. Cells fed with vanadate exhibit a spread morphology, and cells fed with both agents exhibit primarily the spread morphology without much evidence of migration into colonies.

that affects either phosphorylation or dephosphorylation of the single tyrosine acceptor site (tyrosine 1649) in $\beta 4$ protein. Thus, in tumor cells, the tyrosine kinase that phosphorylates tyrosine 1649 may be lost or inactive. There may be a mutation that affects the acceptor site in the $\beta 4$ protein or there may be overexpression or aberrant activity of the protein tyrosine phosphatase that dephosphorylates tyrosine 1649. Kennel et al. [23] have reported that certain lung cancers lack the distal cytoplasmic domain of the $\beta 4$ subunit containing the phosphorylation site. They postulated that this may have functional significance. Preliminary experiments with UM-SCC-38 cells indicate that this is not the case in these cells. Treatment with 3-9-fold higher vanadate concentrations does lead to $\beta 4$ tyrosine phosphorylation and changes in cell morphology similar to those induced in normal keratinocytes at lower concentrations. This implicates increased protein tyrosine phosphatase activity as a basis for the decreased tyrosine phosphorylation of $\beta 4$ observed in squamous cell carcinoma.

Failure to phosphorylate tyrosine or rapid dephosphorylation of tyrosine, in the $\beta 4$ subunit, may be a key difference between normal and malignant keratinocytes. We propose that phosphorylation of tyrosine 1649 may be required for the anchoring process to occur. The location of the $\alpha 6 \beta 4$ integrin in the hemidesmosome in normal skin and mucosa suggests that this integrin is involved in, and perhaps critical to, hemidesmosome assembly. In fact, tyrosine phosphorylation of $\beta 4$ may create a high affinity protein binding site that induces hemidesmosome formation. Since formation of hemidesmosomes may be necessary for differentiation, inhibition of hemidesmosome formation by increased tyrosine phosphatase activity could block differentiation and increase migratory behavior. In normal wound healing this would allow for keratinocyte migration and re-epithelialization; however, in tumor cells, alteration in this process could predispose cells to metastatic behavior. It will be important to determine if integrin phosphorylation can be regulated by chemopreventive agents, since this could inhibit abnormal growth and migration.

\section{ACKNOWLEDGMENTS}

Supported by USPHS grants CA 28564, CA 35929 (TEC), CA 56973 (HBG), PDT-409 and CB-63A from the American Cancer Society (ML), the General Clinical Research Center Grant MO1-RR00042, NIH USPHS T32 HD 07274, and the Department of Veterans Affairs Research Service.

\section{REFERENCES}

1. Kimmel KA, Carey TE, Judd WJ, McClatchey KD: Monoclonal antibody (G10) to a common antigen of human squamous cell carcinoma: Binding of the antibody to the $\mathbf{H}$ type 2 blood group determinant. J Natl Cancer Inst 76:9-16, 1986.

2. Kimmel KA, Carey TE: Altered expression in squamous cells of an orientation restricted epithelial antigen detected by monoclonal antibody A9. Cancer Res 46:3614-3623, 1986.

3. Van Waes C, Kozarsky KF, Warren AB, Kidd L, Paugh D, Liebert M, Carey TE: The A9 antigen associated with aggressive human squamous carcinoma has structural and functional similarity to the newly defined integrin $\alpha 6 \beta 4$. Cancer Res 51:23952402, 1991.

4. Carey TE, Wolf GT, Hsu S, Poore J, Peterson K, McClatchey KD: Expression of A9 antigen and loss of blood group as determinants of survival in patients with head and neck squamous carcinoma. Otolaryngol Head Neck Surg 96:221-230, 1987. 
5. Wolf GT, Carey TE, Schmaltz SP, McClatchey KD, Poore J, Glaser L, Hayashida DJS, Hsu S: Altered antigen expression predicts outcome in squamous cell carcinoma of the head and neck. J Natl Cancer Inst 82:1566-1572, 1990.

6. Carey TE, Van Dyke DL, Worsham MJ, Bradford CR, Babu VR, Schwartz DR, Hsu S, Baker SR: Characterization of human laryngeal primary and metastatic squamous cell carcinoma cell lines UMSCC-17A and UM-SCC-17B. Cancer Res 49:60986107, 1989.

7. Davidsohn I, Norris HJ, Stejskal R, Lill P: Metastatic squamous cell carcinoma of the cervix. Arch Pathol Lab Med 95:132-134, 1973.

8. Limas C, Lange P, Fraley EE, Vasella RL: A, B, and $\mathrm{H}$ antigens in transitional cell tumors of the urinary bladder: Correlation with the clinical course. Cancer 44:2099-2107, 1979.

9. Wolf GT, Carey TE: Tumor antigen phenotype, biologic staging and prognosis in head and neck squamous carcinoma. In "Biology of and Novel Therapeutic Approaches for Epithelial Cancers of the Aerodigestive Tract." J Natl Cancer Inst Monogr, 1992, pp 67-74.

10. Lee JS, Ro JY, Sahin AA, Hong WK, Brown BW, Mountain CF, Hittelman WN: Expression of bloodgroup antigen $\mathrm{A}-\mathrm{a}$ favorable prognostic factor in non-small cell lung cancer. $N$ Engl J Med 324: 1084-1090, 1991.

11. Falcioni R, Kennel SJ, Giacomini P, Zupi G, Sacchi A: Expression of tumor antigen correlated with metastatic potential of Lewis lung carcinoma and B16 melanoma clones in mice. Cancer Res 46:57725778, 1986.

12. Kennel SJ, Foote LJ, Falcioni R, Sonnenberg A, Stringer CD, Crouse C, Hemler ME: Analysis of the tumor-associated antigen TSP-180. Identity with $\alpha 6 \beta 4$ in the integrin superfamily. J Biol Chem 264: 15515-15521, 1989.

13. Falcioni R, Sacchi A, Resau J, Kennel SJ: Monoclonal antibody to human carcinoma-associated protein complex: Quantitation in normal and tumor tissue. Cancer Res 48:816-821, 1988.

14. Kajiji SM, Davcera B, Quaranta V: Six monoclonal antibodies to human pancreatic cancer antigens. Cancer Res 47:1367-1376, 1987.

15. Liebert M, Wedemeyer G, Stein JA, Washington RW Jr, Van Waes C, Carey TE, Grossman HB: The bladder cancer monoclonal antibody BQ16 identifies the $\alpha 6 \beta 4$ integrin. Hybridoma, 1993 (in press).

16. Stepp MA, Spurr-Michaud S, Tisdale A, Elwell J: $\alpha 6 \beta 4$ integrin heterodimer is a component of hemidesmosomes. Proc Natl Acad Sci USA 87:8970-8974, 1990.

17. Sonnenberg A, Calafat J, Janssen H, Daams H, van der Raaij-Helmer LMH, Falcioni R, Kennel SJ, Alpin JD, Baker J, Loizidou M, Garrod D: Integrin $\alpha 6 \beta 4$ complex is located in hemidesmosomes, suggesting a major role in epidermal cell-basement membrane adhesion. J Cell Biol 113:907-917, 1991.

18. Jones JCR, Kurpakus MA, Cooper HM, Quaranta V: A function for the integrin $\alpha 6 \beta 4$ in the hemidesmosome. Cell Regulation 2:427-438, 1991.

19. Kurpakus MA, Quaranta V, Jones JCR: Surface relocation of alpha6 beta4 integrins and assembly of hemidesmosomes in an in vitro model of wound healing. J Cell Biol 115:1737-1750, 1991.

20. Carey TE, Laurikainen L, Nair TS, Reinke TS, Coling DE, Wolf GT, Van Waes C, Liebert M, Marcelo C: Regulation of expression and phosphorylation of $A 9 / \alpha 6 \beta 4$ integrin in normal and malignant keratinocytes. In "Biology of and Novel Therapeutic Approaches for Epithelial Cancers of the Aerodigestive Tract." J Natl Cancer Inst Monogr, 1992, pp 75-86.

21. Riddelle KS, Green KJ, Jones JCR: Formation of hemidesmosomes in vitro by a transformed rat bladder line. J Cell Biol 112:159-168, 1991.

22. Falcioni R, Perrotti N, Piaggio G, Kennel SJ, Sacchi A: Insulin-induced phosphorylation of the beta-4 integrin subunit expressed on murine metastatic carcinoma cells. Mol Carcinogen 2:361-368, 1989.

23. Kennel SJ, Epler RG, Lankford TK, Foote LJ, Dickas V, Canamucio $M$, Cavalierie $R$, Cosimelli $M$, Venturo I, Falcioni R, Sacchi A: Second generation monoclonal antibodies to human integrin $\alpha 6 \beta 4$. Hybridoma 9:243-254, 1990. 